\title{
Mechanical Non Surgical Therapy: An Indispensable Tool
}

\author{
${ }^{1}$ Ashu Bhardwaj, ${ }^{2}$ Ajay Mahajan, ${ }^{3}$ Nisha Thakur, ${ }^{4}$ Naresh Kumar
}

\begin{abstract}
Purpose: Traditional approaches to mechanical debridement of the tooth surface to remove tooth accretions continue to be an integral part of periodontal therapy. The purpose of the present study was to test the effectiveness of mechanical non-surgical therapy in subjects with moderate to severe gingivitis and periodontitis.

Methods: Ten subjects of moderate to severe gingivitis/periodontits were selected for the study. Gingival inflammation, pocket depth and CAL were evaluated from baseline over a period of 8 weeks.

Results: In four subjects with gingivitis the mean gingival inflammation at the base line was $2.15 \pm 0.131 \mathrm{~mm}$ and after 8 weeks of phase-1 periodontal therapy was $1.12 \pm 0.087 \mathrm{~mm}$ with highly statistically significant difference (p-value $=0.000)$. In six periodontitis patients, the mean CAL at the base line was $4.555 \pm 1.461 \mathrm{~mm}$ and after 8 weeks of phase-1 periodontal therapy was $4.302 \pm 1.726 \mathrm{~mm}$. Gain in CAL was $0.254 \pm 0.361 \mathrm{~mm}$ with statistically non-significant difference between the means of CAL at baseline and after 8 weeks ( $p$-value=0.146). In all subjects, the mean pocket depth at the base line was $3.608 \pm 0.738 \mathrm{~mm}$ and after 8 weeks of phase-1 periodontal therapy was $2.795 \pm 1.159 \mathrm{~mm}$ with mean reduction of pocket depth $0.892 \pm 594 \mathrm{~mm}$. The difference was statistically significant ( -value $=0.002$ ).

Conclusions: Non-surgical periodontal therapy induces beneficial changes to periodontal tissues, as expressed by reduction of gingival inflammation, reduction of pocket depth, and gain in clinical attachment level. However, in two subjects with severe periodontitis (mean pocket depth being $5.231 \mathrm{~mm}$ and $4.05 \mathrm{~mm}$ ) the changes were minimal, thus justifying the indication of surgical intervention.
\end{abstract}

Keywords: gingivitis,Non- surgical therapy, periodontitis.

\section{Introduction}

The term periodontal disease in its strictest sense refers to both gingivitis and periodontitis. Gingivitis is an inflammatory condition of the soft tissue surrounding the teeth (the gingiva) and is a direct immune response to the dental microbial plaque building upon teeth. Periodontitis involves the destruction of the supporting structures of the teeth including the periodontal ligament, bone and soft tissues. ${ }^{1}$

From prevalence studies, chronic periodontitis in mild to moderate forms is the most commonly occurring form of periodontitis worldwide ranging in prevalence from $13 \%$ to $57 \%$ in different populations, depending on oral hygiene and socio-economic status. Severe forms of periodontitis affect a minority of a given population and seem to occur in particularly susceptible individuals in proportions not exceeding $10-25 \%$ of population. $^{2}$

The pathogenic processes of the periodontal diseases are largely a result of the host response to microbial induced tissue destruction. These destructive processes are initiated by bacteria but are propagated by host cells. Thus it is the host response that results in tissue destruction. ${ }^{1}$ It is now beyond question that dental plaque is the main etiological factor in the pathogenesis of periodontal disease. ${ }^{3}$ Researches recognise that gingivitis usually does not proceed to periodontitis; however gingivitis frequently precedes periodontitis and usually is associated with periodontitis. ${ }^{4}$ Therefore thorough removal of bacterial deposits from the supra and subgingival environment is the main goal of periodontal therapy. ${ }^{3}$ Elimination of all inflammation remains a critical objective of periodontal therapy. ${ }^{4}$ Successful periodontal therapy is dependent on anti infective procedures acquired at eliminating pathogenic organisms found in dental plaque associated with tooth surface and with in other niches in the oral cavity. ${ }^{5}$ Anti-infective therapy includes both mechanical and chemotherapeutic approaches to minimise or eliminate microbial bio-film(bacterial plaque), the primary aetiology of gingivitis and periodontitis. ${ }^{3}$

Mechanical instrumentation of roots using curettes is an effective treatment for patients with mild to moderate periodontitis. Numerous studies have supported the contention that root planing can reduce probing depths, gain clinical attachments and inhibit disease progression. ${ }^{4} \mathrm{~A}$ number of treatment options are available for the treatment of periodontal disease ranging from the traditional non surgical periodontal therapy(mechanical hand instrumentation, ultrasonic debridement, supra-gingival irrigation, sub-gingival irrigation, local drug delivery, systemic antibiotic therapy and host modulation therapy) to recent surgical treatment modalities utilizing grafts, membranes, growth factors and various other tissue engineered products and techniques. These therapies can be continued with antibiotics and host modulation therapy. Despite various treatment options discussed above, non surgical periodontal therapy still remains the mainstay of the periodontal treatment regimens. ${ }^{6,7,9,10}$ Despite significant advancements in the knowledge of disease pathogenesis and factors 
affecting the progression of the disease, traditional approaches to mechanical debridement of the tooth surface to remove tooth accretions continue to be an integral part of periodontal therapy. ${ }^{11}$ Also nonsurgical treatment is indispensable for many periodontitis patients, especially for those with systemic diseases, such as heart disease and diabetes, who may not be suitable for periodontal surgery. ${ }^{12}$

Keeping all the facts in mind we present a short clinical trial of ten cases treated successfully with non surgical periodontal therapy alone.

\section{Materials and methods}

The present study was conducted in the Department of Periodontology, Himachal Pradesh Govt. Dental College and Hospital, Shimla and was approved by the local ethical committee. Ten subjects coming to the OPD of the department for treatment of moderate to severe gingivitis/moderate to severe periodontitis were selected for the study. Diagnosis of moderate/severe periodontitis were based on the 1999 Consensus Classification of Periodontal Disease by Gary C. Armitage ${ }^{13}$ and by Thomas F. Flemmig (1999). ${ }^{14}$

All subjects signed informed consent. Clinical outcome such as gingival inflammation, pocket depth and CAL were evaluated from baseline over a period of 8 weeks.

Inclusion criteria

- Subjects not older than 50 years of age.

- Subjects willing to participate in the study and sign informed consent.

- Patients having moderate to severe gingivitis/ periodontitis.

- Presence of at least 16 teeth including 4 molar teeth in the oral cavity.

- Good general health of the patient.

Exclusion criteria

- Non compliant subjects.

- Current smokers.

- Pregnant females.

- Subjects having undergone any periodontal treatment in past 6 months.

- Subjects under a long term anti-inflammatory or analgesic medication.

- Medically compromised subjects, in whom periodontal treatment was contraindicated.

Pre-operative assessment

Plaque scores were taken using the Plaque Index (Silness and Loe, 1964) at four designated sites i.e. mesio-facial, mid-facial, disto-facial and lingual. Gingival inflammation was recorded using Gingival Index (Loe and Silness, 1963) at the same four designated sites i.e. mesio-facial, mid-facial, disto-facial and lingual.

Pocket depth was noted using University of North Carolina 15 periodontal probe on mesio-facial, midfacial, disto-facial and lingual surfaces rounding off to the nearest higher millimetre, by an examiner.

Clinical attachment levels were also noted at the four designated sites to the nearest highest millimetre, by calculating the distance from free gingival margin (FGM) to the base of the pocket. Following baseline measurements of all the subjects received Phase-1 periodontal treatment using piezoelectric ultrasonic scaler and hand instruments. The subjects received oral hygiene instructions including tooth brushing and inter-dental cleaning according to the individual needs. Patients were recalled for follow-up one week after Phase-1 periodontal therapy, and follow-up appointments were scheduled every two weeks till the end of the study. At the end of 8 weeks, the clinical parameters measured at baseline were re-recorded, to evaluate the treatment outcomes.

In statistical analysis, the value of continuous variables was expressed as mean $\pm \mathrm{SD}$ (standard deviation). The significance of difference in mean values of continuous variables before and after phase-1 periodontal therapy was estimated by student's $\mathrm{t}$ - test (paired samples T-test and one sample T-test). Two tailed p-value of $<0.05$ was taken as statistically significant.

\section{Results}

At the end of the study, the mean age of the subjects was $29.1 \pm 10.005$ years with a range of 13-48 years and $40 \%$ of the subjects were with moderate to severe gingivitis and $60 \%$ of the subjects were with moderate to severe periodontitis with highest score of gingival inflammation 2.287 and maximum loss of clinical attachment level was in the range of 6.566 . The following results were obtained (Table $1,2 \& 3$ ) by using computerised statistical software (SPSS-16.0). 


\section{Discussion}

The present study was conducted in the department of Periodontology, Himachal Pradesh Govt. Dental College and Hospital, Shimla on ten subjects having moderate to severe gingivitis and periodontitis. The study was designed to assess the effectiveness of non-surgical periodontal therapy on the severity of periodontal disease. The aim of this non surgical periodontal therapy was to eliminate both living bacteria in the microbial biofilm and calcified biofilm microorganisms' i.e. dental calculus, from the root surfaces without the surgical reflection of the soft tissues surrounding the teeth resulting in reduction in number of biofilm microorganisms and a disturbance in the ecology of the microbial biofilm. As a result, host will be able to better control the microbial recolonisation of the dento-gingival area by personal oral hygiene measures. ${ }^{10}$

Bleeding on probing is present during the active phase of periodontitis which means that sulcular/pocket epithelium is no longer intact, being more permeable to lipopolysaccharides (LPS) and other bacterial products. The gram negative anaerobic infection is postulated to provide a reservoir of lipopolysaccharides that can trigger mediators of inflammation such as interleukin-1 $\beta$ (IL-1 $\beta$ ), tumor necrosis factor-alpha (TNF-alpha) and prostaglandin $\mathrm{E}_{2}\left(\mathrm{PGE}_{2}\right)$ resulting in increase in severity of gingival inflammation. ${ }^{15}$ Non surgical periodontal therapy leads to remarkable decrease in severity of gingival inflammation by decreasing the amount of bacteria and their products in the gingival sulcus. ${ }^{10,12}$ In the present study, the mean gingival inflammation at the base line was $2.15 \pm 0.131 \mathrm{~mm}$ and after 8 weeks of phase- 1 periodontal therapy was $1.12 \pm 0.087 \mathrm{~mm}$ (Table-1). The difference in means of gingival inflammation at baseline and after 8 weeks was statistically highly significant ( $p$-value $=0.000$ ) thereby establishing the fact that moderate to severe gingivitis patients can be treated successfully with the phase-1 periodontal therapy alone.

Although CAL does not yield any data on the present activity of periodontitis, CAL only shows that periodontitis is present but not necessarily in active phase. Therefore we used it as to present the amount of mean periodontal destruction on subjects with moderate to severe periodontitis and further any change in CAL (either gain or loss in CAL) after phase-1 periodontal therapy. In the present study, the mean CAL at the base line was $4.555 \pm 1.461 \mathrm{~mm}$ and after 8 weeks of phase- 1 periodontal therapy was $4.302 \pm 1.726 \mathrm{~mm}$ with gain in CAL $0.254 \pm 0.361 \mathrm{~mm}$ (Table-2). The difference was not statistically significant between the means of CAL at baseline and after 8 weeks ( $\mathrm{p}$-value $=0.146$ ). Out of six subjects with moderate to severe periodontitis, in four subjects there was gain in CAL with two subjects (mean pocket depth being $5.231 \mathrm{~mm}$ and $4.05 \mathrm{~mm}$ ) having loss in CAL 8 weeks after phase-1 periodontal therapy and these 2 subjects were having severe form of periodontitis indicating that phase-1 periodontal therapy has less pronounced effect in patients with severe periodontitis.

The pocket depth gives no information on the extent and severity of the periodontitis but simply registers the current situation, and as such cannot be considered representative information on the disease history and extent as stated by Apatzidou and Kinane in 2004. ${ }^{16,17}$ The surface area of the gingiva where bacteria can invade/ diffuse the tissues is larger if the probing depth is greater (Radnai et $\mathrm{al}^{15}$ ). So by reduction in pocket depth we can achieve periodontal health. In the present study, the mean pocket depth at the base line was $3.608 \pm 0.738 \mathrm{~mm}$ and after 8 weeks of phase-1 periodontal therapy was $2.795 \pm 1.159 \mathrm{~mm}$ with mean reduction of pocket depth $0.892 \pm 594 \mathrm{~mm}$ (Table-3). The difference was statistically significant between the means of pocket depth score at baseline and after 8 weeks ( $p$-value $=0.002$ ) indicating that mechanical non-surgical therapy provide an indispensable tool for pocket depth reduction. Also this probing pocket depth reduction is beneficial since it results in an environment that is less favourable for the establishment of anaerobic periodontopathic microorganisms leading the periodontal tissues towards health. ${ }^{10}$

It can be concluded from this clinical trial that in spite of various current treatment modalities, non-surgical periodontal therapy is the integral part of periodontal therapy which induces beneficial changes to the periodontal tissues, as expressed by a reduction of the gingival inflammation, reduction of probing pocket depth, and gain in clinical attachment level. The changes are remarkable in subjects with moderate to severe gingivitis and moderate periodontitis, but minimal in subjects with severe periodontitis.

\section{Acknowledgments -}

This study was supported by a grant from Govt. of Himachal Pradesh. The authors appreciate the cooperation of the patients during the course of this study.

\section{References}

[1] Kinane DF. Causation and pathogenesis of periodontal disease. Periodontology 2000, Vol. 25, 2001, 8-20

[2] Rylev M, Kilian M. Prevalence and distribution of principal periodontal pathogens worldwide. J Clin Periodontol 2008 ; 35 (Suppl. 8): 346-361.

Ishikawa I, Baehni P. Nonsurgical periodontal therapy - where do we stand now? Periodontology 2000, Vol. 36, $2004,9-13$.

Greenstein G, Nonsurgical periodontal therapy in 2000: A literature review. J Am Dent Assoc 2000;131;1580-1592.

Drisko CH. Nonsurgical periodontal therapy. Periodontology 2000, Vol. 25, 2001, 77-88.

Cobb CM. Non-surgical pocket therapy: mechanical. Ann Periodontol 1996: 1: 443-490.

Cobb CM. Clinical significance of non-surgical periodontal therapy: an evidence-based perspective of scaling and root planing. $J$ Clin Periodontol 2002: 29 (Suppl. 2): 6-16. 
[8] Cobb CM. Oral biofilms in health and disease. In Newman HN, Wilson M, ed. The mechanical control of subgingival plaque. Dental plaque revisited. London, Eastman Dental Institute, University College, 1999: 457-502.

[9] Greenstein G. Periodontal response to mechanical non-surgical therapy: a review. J Periodontol 1992; 63(2): 118-30.

[10] Adriaens PA, Adriaens LM. Effects of nonsurgical periodontal therapy on hard and soft tissues. Periodontology 2000, Vol. 36, 2004, 121-145

[11] Suvan JE. Effectiveness of mechanical non-surgical pocket therapy. Periodontology 2000, Vol. 37, $2005,48-71$.

[12] Umeda M, Takeuchi Y, Noguchi K, Huang Y, Koshy G and Ishikawa I. Effects of nonsurgical periodontal therapy on the microbiota. Periodontology 2000, Vol. 36, 2004, 98-120.

[13] Armitage GC. Consensus report: chronic periodontitis. Ann Periodontol 1999; 4: 38.

[14] Flemmig TF. Periodontitis. Ann Periodontol 1999; 4: 32-37.

[15] Radnai M, Gorzo I, Nagy E, Urban E, Novak T, Pal A. A possible association between preterm birth and early periodontitis. J Clin Periodontol 2004; 31: 736-741.

[16] Bosnjak A, Relja T, Vucicevic-Boras V, Plasaj H, Plancak D. Pre-term delivery and periodontal disease:a case-control study from Croatia. J Clin periodontal 2006; 33: 710-716.

[17] Apatzidou DA, Kinane DF. Quadrant root planing versus same-day full- mouth root planning. Journal of Clin Periodontol 2004; 31:132-140.

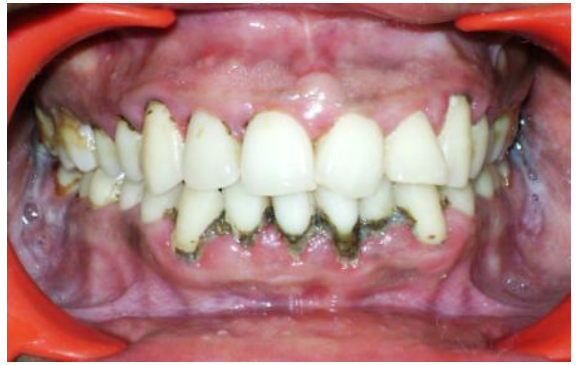

Figure No. 1 showing moderate gingivitis before non surgical therapy.

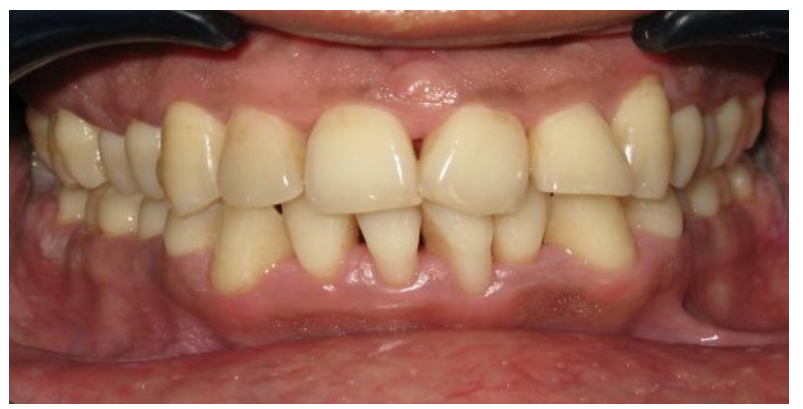

Figure No. 2 showing healthy gingiva after non surgical therapy.

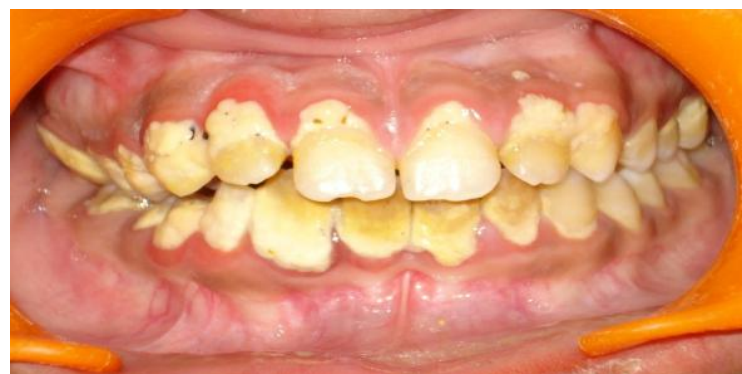

Figure No. 3 showing severe gingivitis before non surgical therapy.

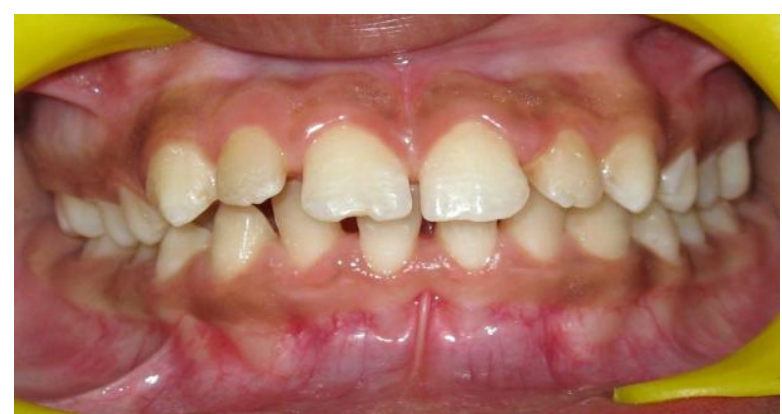

Figure No. 4 showing healthy gingiva after non surgical therapy. 


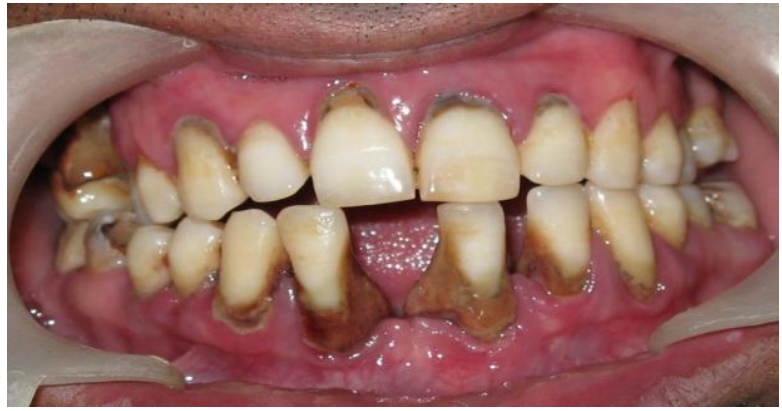

Figure No. 5 showing moderate periodontitis before non surgical therapy.

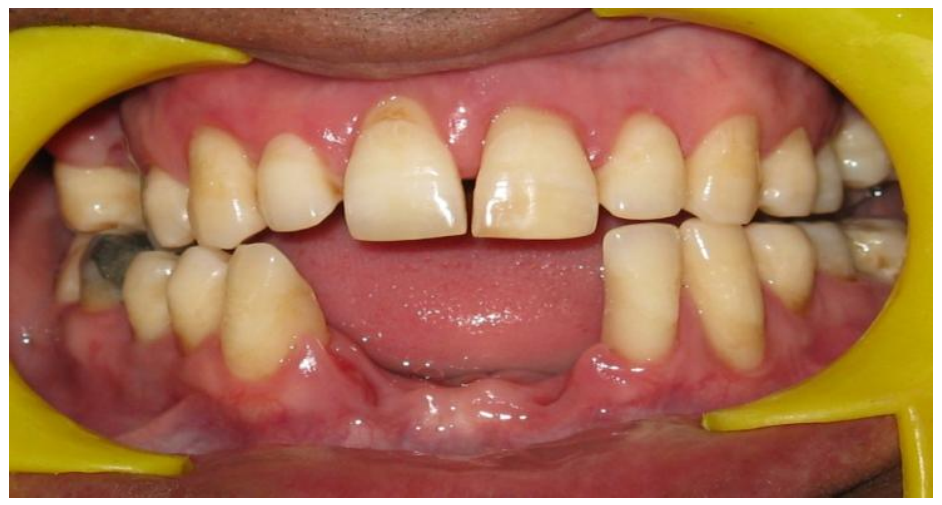

Figure No. 6 showing healthy periodontium after non surgical therapy.

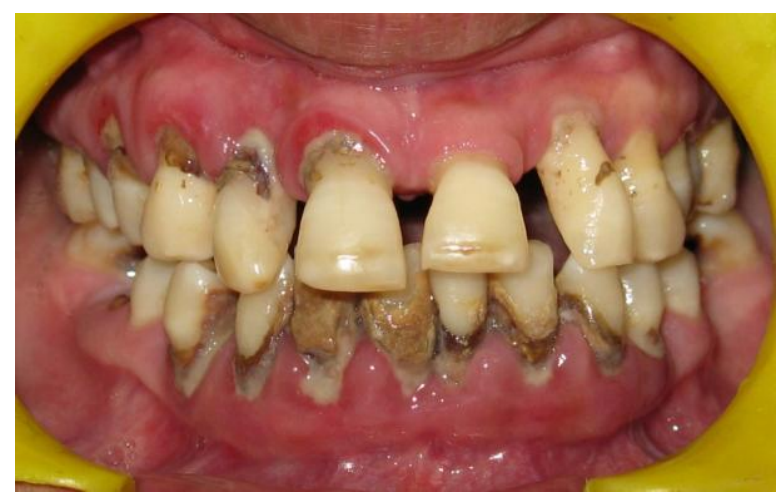

Figure No. 7 showing severe periodontitis before non surgical therapy.

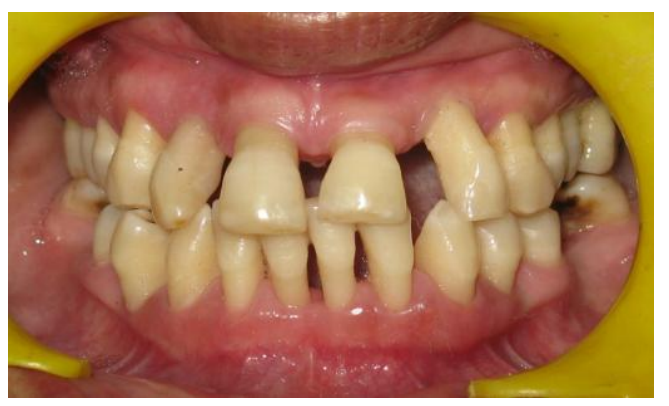

Figure No. 8 showing healthy periodontium after non surgical therapy.

Table.1. showing the results of Phase-1 periodontal therapy in moderate to severe gingivitis patients.

\begin{tabular}{|l|l|l|l|}
\hline $\begin{array}{l}\text { Patients with } \\
\text { moderate/severe } \\
\text { gingivitis }\end{array}$ & $\begin{array}{l}\text { Gingival inflammation score } \\
\text { at baseline }(\text { Mean } \pm \text { SD) }\end{array}$ & $\begin{array}{l}\text { Gingival inflammation } \\
\text { Score at 8 weeks (Mean } \pm \\
\text { SD) }\end{array}$ & p-value \\
\hline 4 & $2.15 \pm 0.136$ & $1.12 \pm 0.087$ & $0.000^{*}$ \\
\hline
\end{tabular}


Mechanical Non Surgical Therapy:An Indispensable Tool (A Short Clinical Trial)

Table.2. showing the results of Phase-1 periodontal therapy in moderate to severe periodontits patients.

\begin{tabular}{|l|l|l|l|l|}
\hline $\begin{array}{l}\text { Patients with } \\
\text { moderate/severe } \\
\text { periodontits }\end{array}$ & $\begin{array}{l}\text { Clinical attachment } \\
\text { level loss at } \\
\text { baseline (Mean } \pm \\
\text { SD) }\end{array}$ & $\begin{array}{l}\text { Clinical attachment } \\
\text { level loss at 8 } \\
\text { weeks } \\
\text { (Mean } \pm \text { SD) }\end{array}$ & $\begin{array}{l}\text { Gain in clinical } \\
\text { attachment level } \\
\text { (Mean } \pm \text { SD) }\end{array}$ & p-value \\
\hline 6 & $4.555 \pm 1.461$ & $4.302 \pm 1.726$ & $0.254 \pm 0.361$ & 0.146 \\
\hline
\end{tabular}

Table.3. showing the results of Phase-1 periodontal therapy on pocket depth reduction in moderate to severe gingivitis and periodontits patients.

\begin{tabular}{|l|l|l|l|l|}
\hline $\begin{array}{l}\text { Patients with } \\
\text { moderate/severe } \\
\text { gingivitis/Periodontitis }\end{array}$ & $\begin{array}{l}\text { Pocket depth score } \\
\text { at baseline } \\
\text { (Mean } \pm \text { SD) }\end{array}$ & $\begin{array}{l}\text { Pocket depth } \\
\text { score at 8 weeks } \\
\text { (Mean } \pm \text { SD) }\end{array}$ & $\begin{array}{l}\text { Pocket depth } \\
\text { reduction (Mean } \\
\pm \text { SD) }\end{array}$ & P-value \\
\hline 10 & $3.608 \pm 0.738$ & $2.795 \pm 1.159$ & $0.892 \pm 594$ & $0.002 *$ \\
\hline
\end{tabular}

